\title{
The peak flux distribution of bright gamma-ray bursts measured with ULYSSES
}

\author{
J.-L. Atteia ${ }^{1}$, M. Boër ${ }^{1}$, and K. Hurley ${ }^{2}$ \\ 1 Centre d'Étude Spatiale des Rayonnements, CNRS/UPS, BP. 4346, 31028 Toulouse Cedex 4, France \\ e-mail: atteia@cesr.fr, boer@cesr.fr \\ 2 University of California at Berkeley, Space Sciences Laboratory, Berkeley, CA 94720-7450, U.S.A. \\ e-mail: khurley@sunspot.ssl.berkeley.edu
}

Received January 21; accepted May 28, 1999

\begin{abstract}
Observations of gamma-ray burst afterglows have shown that these events come from formidable explosions at cosmological distances. If the most distant GRBs seen by BATSE have redshifts of the order of $3-6$ (e.g. GB 971214), it is a coincidence that the bright end of the GRB intensity distribution displays the $-3 / 2$ slope expected for a Euclidean distribution of sources. We analyze nearly 10 years of continuous observation of bright GRBs with ULYSSES, in order to constrain their intensity distribution. This extensive dataset (more than 200 bursts in the "Euclidean" part of the curve) is used to quantify the agreement of the data with the canonical slope of $-3 / 2$.
\end{abstract}

Key words: gamma-rays: bursts

\section{Introduction}

The GRB detector onboard ULYSSES has been working almost continuously since it was turned on, on November 9, 1990. ULYSSES presents several characteristics that are of interest for the study of the brightness distribution of the rare, intense GRBs: continuous operation for several years, an almost unobstructed field of view and a constant energy threshold. In addition, the GRB detector is small, making it mostly insensitive to dead time and pileup effects that affect the study of very bright bursts with larger instruments. Finally, in April 1998, the sky coverage of ULYSSES amounted to $87.4 \mathrm{yr}$ sr, a number which is comparable to the sky coverage of PVO over its entire lifetime $(\sim 125 \mathrm{yr}$ sr, Fenimore et al. 1993).

We present here the peak flux distribution of 331 GRBs detected by ULYSSES from November 1990 to April 1998. The burst intensity is measured by the peak count rate

Send offprint requests to: J-L. Atteia over $1 \mathrm{~s}$ (hereafter $C_{\max }$ ), within the full ULYSSES energy range (approximately $20-150 \mathrm{keV}$ ). In order to facilitate the comparison with BATSE we also indicate the corresponding $50-300 \mathrm{keV}$ peak flux ${ }^{1}$. Finally, we have restricted our analysis to GRBs with durations $>2 \mathrm{~s}$.

We study 2 samples that we describe now.

Triggers: The GRBs that triggered ULYSSES. The sky coverage of ULYSSES for these bursts is $87.4 \mathrm{yr}$ sr. This sample is complete down to $9 \mathrm{ph} \mathrm{cm}^{-2} \mathrm{~s}^{-1}\left(C_{\max }=\right.$ $250 \mathrm{~s}^{-1}$ ) and contains 168 GRBs above this threshold.

Real Time events: The GRBs found in ULYSSES real time data from the Current BATSE Catalog. Starting from the BATSE Catalog allows us to use GRBs that are a factor of 2.5 fainter at the price of reducing the spatial coverage by a factor of 2 . The sky coverage of ULYSSES for these bursts is $42.2 \mathrm{yr}$ sr. This sample is complete down to $3.6 \mathrm{ph} \mathrm{cm}^{-2} \mathrm{~s}^{-1}\left(C_{\max }=100 \mathrm{~s}^{-1}\right)$ and contains 331 GRBs above this threshold.

\section{Results}

The peak flux distributions for the 2 samples are displayed in Fig. 1. The slopes of the observed distributions appear compatible with the value expected for sources homogeneously distributed in Euclidean space (indicated by dotted lines). In order to obtain a more quantitative statement we have computed the maximum likelihood estimate of the slope $(s)$ and the probability $\left(P_{-3 / 2}\right)$ for the data to come from a "Euclidean" distribution. $P_{-3 / 2}$ is computed with a Kolmogorov-Smirnov test.

$$
\begin{aligned}
& * \text { Triggers: } s=-1.59 \pm 0.11 \quad\left(P_{-3 / 2}=0.48\right) . \\
& * \text { Real time GRBs: } s=-1.41 \pm 0.07 \quad\left(P_{-3 / 2}=0.12\right) .
\end{aligned}
$$

\footnotetext{
${ }^{1}$ On average a $50-300 \mathrm{keV}$ peak flux of $1 \mathrm{ph} \mathrm{cm}^{-2} \mathrm{~s}^{-1}$ corresponds to 28 counts in ULYSSES.
} 


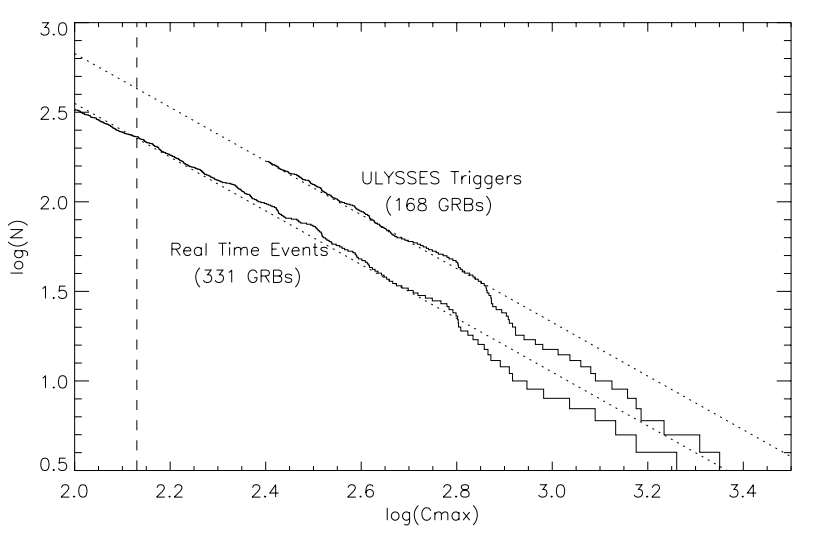

Fig. 1. The GRB peak flux distribution measured by the gamma burst detector on ULYSSES (see text). The dotted lines have the slope $-3 / 2$ expected for a Euclidean distribution

* Real time GRBs with a peak flux $\geq 5 \mathrm{ph} \mathrm{cm}^{-2} \mathrm{~s}^{-1}$ (231 bursts): $s=-1.51 \pm 0.10 \quad\left(P_{-3 / 2}=0.81\right)$

(this threshold is indicated by the vertical line in Fig. 1.)

We conclude that the peak flux distribution of GRBs with $P \geq 5 \mathrm{ph} \mathrm{cm}^{-2} \mathrm{~s}^{-1}\left(C_{\max } \geq 135 \mathrm{cs}^{-1}\right)$ measured by ULYSSES shows no deviation from the "Euclidean" case. This does not necessarily imply that the true peak flux distribution is a power law (a smoothly changing slope could also accomodate the data). This is rather an indication that the deviations we measure from the "Euclidean" case are fully compatible with random fluctuations. Even if ULYSSES provides the best constraints to date on the slope of the bright end of the distribution, it cannot constrain it beyond the quoted value of $-1.51 \pm 0.10$. Combining ULYSSES and PVO data could probably reduce the uncertainty on this number. However, this is difficult due to the almost non-overlapping energy ranges of these 2 instruments.

A careful computation of the ULYSSES livetime for GRB detection allows us to measure the rate of "Euclidean GRBs" with a good confidence. We find that they occur at a rate of $\sim 65 \mathrm{yr}^{-1}$ (for GRBs longer than 2 seconds).

\section{Discussion}

A remarkable step forward has been achieved recently with the discovery of the GRB afterglow emission (Costa et al. 1997; Van Paradijs et al. 1997) which made it possible to measure the redshifts of some GRB hosts. In Fig. 2 we show, on top of the ULYSSES peak flux distribution, the peak fluxes of $8 \mathrm{GRBs}$ with known host redshifts. It is interesting to note that the "Euclidean" region of the peak flux distribution contains at least one, and possibly two, very distant sources (GB 971214 and GB 980329). If future observations confirm that a significant fraction of bright GRBs come from sources with redshifts $>1$, this might be an indication that GRBs undergo significant luminosity evolution.

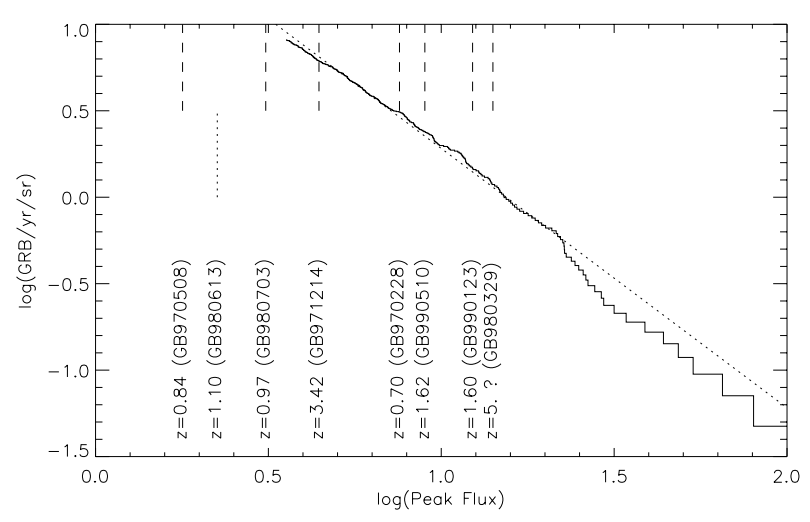

Fig. 2. The peak flux distribution of ULYSSES real time GRBs in physical units. Also indicated are the peak fluxes of 8 GRBs with measured redshifts (the value for GB 980613 is an upper limit as this burst is not seen in ULYSSES real time data)

Our observations raise the issue of the interpretation of the $-3 / 2$ slope measured for bright bursts. Until the beginning of the $90^{\prime} \mathrm{s}$ it was generally thought that this slope reflected the spatial homogeneity of nearby bursters in our local Euclidean universe. With the few redshifts now available, it appears that this explanation is no longer tenable. It is clear now that GRBs have a broad luminosity function, that they undergo significant density evolution (if they are the result of stellar evolution the burst rate must follow the stellar formation rate) and possibly luminosity evolution. In such a complex situation the interpretation of the peak flux distribution requires at least some knowledge of these effects. Understanding the influence of the luminosity function and of the density and luminosity evolution will probably require the measure of a few tens of redshifts. Until then the interpretation of the slope at the bright end of the peak flux distribution will remain speculative.

On the other hand, when we understand the role of the various parameters that impact the shape of the peak flux distribution, it will become increasingly important to have homogeneous datasets containing hundreds of GRBs like those of ULYSSES and BATSE. The long observing time of these two missions and the fact that they have hundreds of GRBs in common offer the opportunity to construct number-intensity curves with unprecedented accuracy over a broad range of intensities. In this respect it is especially important to continue to accumulate as much data as possible with these instruments.

Acknowledgements. We are grateful to the BATSE team for making the Current BATSE GRB Catalog available. $\mathrm{KH}$ is grateful for support under JPL Contract 958056 and NASA grant NAG5-1560.

\section{References}

Costa E., et al., 1997, Nat 387, 783

Fenimore E.E., et al., 1993, Nat 366, 40

van Paradijs J., et al., 1997, Nat 386, 686 